\title{
Factores de riesgo cardiovascular en mayores de 80 años
}

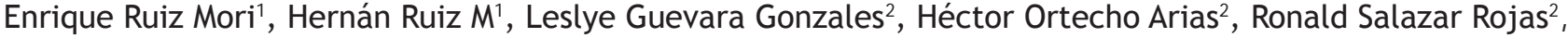 \\ Cristina Torres Mallma², Cindy Vasquez Tornero²,
}

\section{RESUMEN}

Objetivo: En el Perú, la población mayor de 80 años se está incrementando y las enfermedades cardiovasculares son su principal causa de muerte. El objetivo del estudio fué analizar los factores de riesgo cardiovascular en octogenarios. Material y Métodos: Es una investigación observacional, descriptivo, de prevalencia, transversal, realizado en marzo 2015 en Lima Metropolitana. Se utilizó un cuestionario sobre factores de riesgo cardiovascular; se registró la presión arterial, peso, talla y el índice masa corporal, en mayores de 80 años.

Resultados: Se evaluaron a 969 sujetos, de los cuales 562 (58\%) fueron mujeres y 407 (42\%) eran hombres; con edad promedio de 84.2 años; predominó el grupo etario de 80 a 84 años en un 60,5\%. 427 eran hipertensos (44,1\%), y era más común en mujeres $(62,2 \%)$. El $9 \%$ de la población estudiada (87 casos) eran fumadores; siendo más habitual en hombres $(64 \%)(p=0.000009)$. Se registraron a 220 sujetos $(22.7 \%)$ con hipercolesterolemia, siendo más frecuente en mujeres $(139$ pacientes: $63,2 \%$ ), sin significancia estadística. La diabetes se reportó en el $11,5 \%$ de la muestra estudiada (111 pacientes), siendo la frecuencia mayor en mujeres $(68,5 \%)(\mathrm{p}=0.018)$. Según los valores del IMC, 537 sujetos $(55.4 \%)$ tuvieron un IMC $<25$, mientras que el $33,8 \%$ de la población (328) tenían sobrepeso y un 10,7\% cursaban con obesidad, prevaleciendo más en mujeres $(70,2 \%)(p=0.028)$. En la población hipertensa el $87 \%$ se encontraba en tratamiento farmacológico, de los cuales el 65\% estaban controlados. El 26.5\% (257 casos) de la población estudiada tenían dos factores de riesgo y el 13.1\% (127) tres o más factores de riesgo.

Conclusión: El factor de riesgo cardiovascular más frecuente ha sido la Hipertensión Arterial, predominando en mujeres.El $40 \%$ de los sujetos evaluados tenían dos o más factores de riesgo.El 87\% de los pacientes hipertensos recibían tratamiento farmacológico y el 65\% de ellos estaban controlados (Horiz Med 2015; 15(3): 26-33)

Palabras clave: Riesgo cardiovascular, Factores de riesgo, Enfermedad coronaria isquémica, Adulto mayor (Fuente: DeCS BIREME).

\section{Cardiovascular risk factors in adults 80 years of age or older}

\section{ABSTRACT}

Objective: In Peru, the 80 year old and older population is increasing and cardiovascular diseases are the leading cause of death. The aim of the study was to analyze the cardiovascular risk factors in octogenarians.

Material and Methods: This is a descriptive, observational, cross prevalence research, conducted in March 2015 in Lima. A questionnaire on cardiovascular risk factors was used; blood pressure, weight, height and body mass index, in people 80 years of age or older.

Results: 969 subjects were evaluated, of whom $562(58 \%)$ were women and 407 (42\%) were male; with an average age of 84.2 years; predominant age group of $80-84$ years $60.5 \%$. 427 cases were hypertensive $(44.1 \%)$, this was more common in women $(62.2 \%) .9 \%$ of the study population (87 cases) were smokers; being more common in men $(64 \%)(p=0.000009)$. 220 subjects $(22.7 \%)$ with hypercholesterolemia were registered, this being more common in women; 139 patients $(63.2 \%)$, without statistical significance. Diabetes was reported in $11.5 \%$ of the studied sample (111 patients) and it was more frequent in women $(68.5 \%)(p=0.018)$. According to BMI values, 537 subjects $(55.4 \%)$ had a $B M I<25$, while $33.8 \%$ of the population (328) were overweight and $10.7 \%$ were obese, which was more prevalent in women $(70,2 \%)(p=0.028)$. In the hypertensive population, $87 \%$ had drug treatment, of which $65 \%$ were controlled. $26.5 \%$ (257 cases) had two risk factors and $13.1 \%$ (127) had three or more risk factors.

Conclusion: The most frequent factor of cardiovascular risk has been hypertension, predominantly in women. 40\% of the evaluated subjects had two or more risk factors. $87 \%$ of hypertensive patients received drug treatment and $65 \%$ of them were controlled. (Horiz Med 2015; 15(3): 26-33)

Key words: Cardiovascular Risk, Risk Factors, Ischemic Heart Disease, Elderly Population. (Source: MeSH NLM).

1 Profesor de Fisiología y Fisiopatología. Facultad de Medicina Universidad de San Martín de Porres. Lima-Perú

2 Alumnos de la Facultad de Medicina USMP. 


\section{INTRODUCCIÓN}

A nivel mundial la población geronte está experimentando un crecimiento demográfico raudo.

En USA el año 2000 , el $12 \%$ de la población era mayor de 65 años y se espera que para el 2030 la cifra será del $20 \%(1)$.

El Perú, no es ajeno a esta realidad, en el año 2014, las personas mayores de 65 años representaban el $6,4 \%$ de la población total ( $1^{\prime} 972,107$ personas) y los octogenarios de 146,378 en el año 1995 (0,61\%) aumentaron a 367,400 en el $2015(1,1 \%)$ y se estima que alcanzarán la cifra de 551,817 en el año 2025 $(1,6 \%)(2,3)$.

Sólo Lima Metropolitana cuenta en la actualidad con una población mayor de 80 años de 148,797 sujetos (3).

Este incremento en la expectativa de vida de la población, se acompaña de un enorme consumo de servicios de salud que la mayoría de países no están preparados para afrontar, pudiendo generar importantes crisis económicas, de recursos médicos y éticos.

En sujetos mayores de 80 años, las enfermedades cardiovasculares (ECV) son la principal causa de muerte y/o discapacidad, y genera una carga económica cuantiosa (4).

En el Perú, producto del crecimiento económico y de la mejora de su Sistema de Salud, hay una transición demográfica y epidemiológica, que está desplazando las enfermedades infecto-contagiosas por las ECV, como principal causa de muerte en la población adulta(5).

En este contexto, la Enfermedad Coronaria Isquémica $(\mathrm{ECl})$ se ubica como la segunda causa de mortalidad con una tasa de 251 por 100 mil habitantes, en un cuarto lugar se encuentra la enfermedad hipertensiva $(190,7$ por $100 \mathrm{mil})$, seguida por la enfermedad cerebrovascular $(184,9$ por $100 \mathrm{mil}$ (6); al agrupar estas entidades nosológicas bajo el título de ECV se objetiva su trascendencia como causa de muerte en la población peruana. Al analizar los principales factores que propician estas enfermedades se puede establecer que son producto principalmente de una sociedad que se desenvuelve en un medio caracterizado por el mayor número de fumadores, o de mayor ingesta de dietas hipercalóricas, o con un ritmo de vida sedentario $\mathrm{y} / \mathrm{o}$ que se desarrolla en un medio estresante; situaciones que conllevan al desarrollo de la enfermedad coronaria isquémica $(\mathrm{ECl})$ y la hipertensión arterial (HTA) en la comunidad (7).

Una forma de contribuir a la reducción de la mortalidad cardiovascular en mayores de 80 años, es actuando sobre los factores de riesgo que determinan la enfermedad coronaria, más aun tratándose de un proceso altamente prevenible.

Sin embargo; las políticas de prevención en ancianos es escasa, con poca evidencia científica sobre promoción de la salud en dicha población.

El objetivo principal del estudio fué analizar la prevalencia y las características de los factores de riesgo cardiovascular en una población citadina mayor de 80 años, para contar con una herramienta que ayude a la toma de futuras decisiones.

\section{MATERIAL Y MÉTODOS}

Investigación epidemiológica, observacional, descriptiva, de prevalencia, transversal, realizada en el mes de marzo del 2015 en la ciudad de Lima y la Provincia Constitucional del Callao.

Se estructuró un cuestionario para obtener los datos sobre presión arterial, tabaquismo, hipercolesterolemia, diabetes y obesidad; en sujetos mayores de 80 años.

Participaron 380 alumnos del tercer año de medicina de la Universidad de San Martín de Porres, quienes fueron entrenados para el registro de la presión arterial, utilizando la Guía del JNC VII (8),para la clasificación de la presión; así mismo para la determinación del índice masa corporal (IMC) se realizó bajo las normas de la Organización Mundial de la Salud (OMS) (9). 
Todos los participantes firmaron un consentimiento antes de responder al cuestionario y ser evaluados. Se utilizó el paquete R Statistics versión 2.13.0 para Windows.

\section{RESULTADOS}

La población estudiada estuvo constituida por 969 sujetos, de los cuales 562 (58.0\%) fueron mujeres y 407 (42.0\%) hombres; con una edad promedio de 84.2 años (rango, 80 a 102 años), no existiendo diferencia significativa entre los géneros ( 84,1 años en mujeres y 84,2 en varones).

Predominó el grupo etario de 80 a 84 años $(60,5 \%)$. Los mayores o iguales a 90 años, se encontraron en un $11,2 \%$ (Tabla $\mathrm{N}^{\circ}$ 1).

Tabla 1. Distribución por grupos de edad según género

\begin{tabular}{lccc} 
& \multicolumn{3}{c}{ Género $(\%)$} \\
& Mujeres & Hombres & Total \\
Grupos de edad & & & \\
$80-84$ & 333 & 253 & 586 \\
$85-89$ & 169 & 105 & 274 \\
$\geq 90$ & 60 & 49 & 109 \\
Total & $562(58.0)$ & $407(42.0)$ & $969(100.0)$ \\
& & &
\end{tabular}

$X 2=2.244, p=0.326$

Con relación a la presión arterial: 542 sujetos $(55,9 \%)$ eran normotensos predominando en el género masculino $(60,4 \%)$; se halló HTA en el $44,1 \%$ (427 casos) y fué más frecuente en las mujeres $(62,2 \%)$.

El 9\% de la población estudiada (87 casos) eran sujetos fumadores; siendo más frecuente en los hombres (64\%).

Encontrándose una asociación significativa entre el hábito de fumar y el género $(X 2=19.627, p=$ 0.000009).
Según el nivel de colesterol 220 sujetos (22.7\%) tenían hipercolesterolemia, siendo más frecuente esta condición en mujeres $(63,2 \%)$, sin significancia estadística.

La diabetes, se reportó en el $11,5 \%$ de la muestra estudiada (111 pacientes) y fue más frecuente en el sexo femenino al registrarse $76(68,5 \%)$.

Existiendo asociación significativa entre la diabetes $y$ el género $(X 2=5.642, p=0.018)$.

Según los valores del IMC, 537 sujetos (55.4\%) tuvieron un IMC menor de 25, mientras que el 33,8\% de la población (328 sujetos) tenían sobrepeso y un $10,7 \%$ cursaban con obesidad (104 casos); con mayor prevención en las mujeres $(70,2 \%$ contra $29,8 \%)$, existiendo asociación significativa $(X 2=$ 7.153, $\mathrm{p}=0.028$ ) Tabla 2.

La población hipertensa, predominó en el grupo etario de 80 a 84 años con 246 casos (57,6\%), y en todos los grupos fue más frecuente la HTA en las mujeres que en los hombres, pero sin asociación significativa entre los grupos de edad y el género (Figura 1).

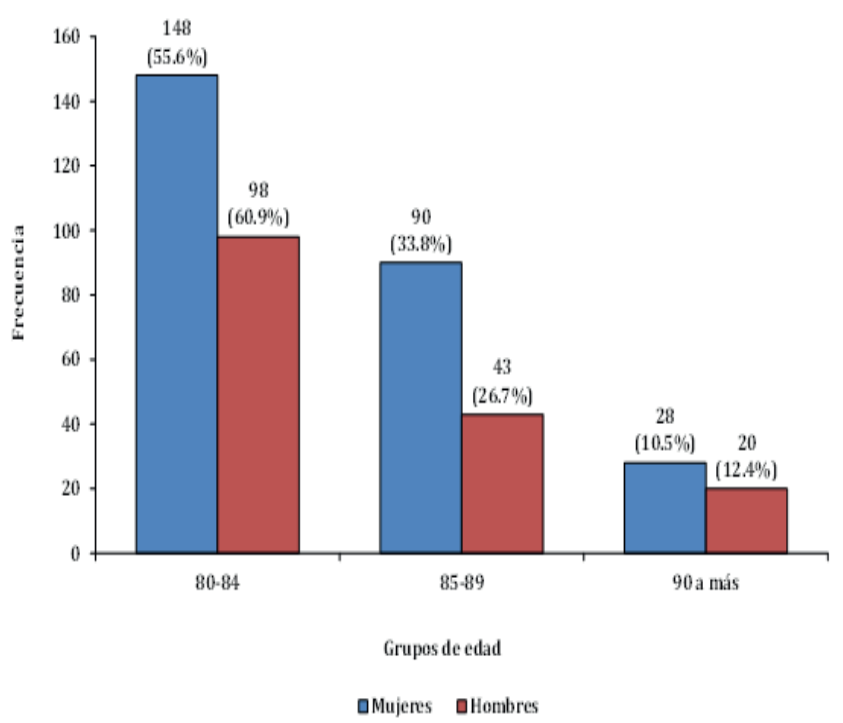

Figura 1. Distribución por grupos de edad según género en pacientes con antecedentes de hipertensión arterial. 
Tabla 2. Prevalencia de factores de riesgo cardiovascular en adultos mayores de 80 años por grupo etario y género

\begin{tabular}{|c|c|c|c|}
\hline Factor de riesgo & Total & Hombres & Mujeres \\
\hline & $\mathbf{N}(\%)$ & $\mathbf{N}(\%)$ & $\mathbf{N}(\%)$ \\
\hline Hipertensión arterial & $427(44.1 \%)$ & $161(37.8 \%)$ & $266(62.2 \%)$ \\
\hline 80 - 84 años & $246(57.6 \%)$ & $98(60.9 \%)$ & $148(55.6 \%)$ \\
\hline 85 - 89 años & $133(31.1 \%)$ & $43(26.7 \%)$ & $90(33.8 \%)$ \\
\hline 90 años a más & $48(11.2 \%)$ & $20(12.4 \%)$ & $28(10.5 \%)$ \\
\hline Tabaquismo* & $87(9.0 \%)$ & $56(64.0 \%)$ & $31(36.0 \%)$ \\
\hline 80 - 84 años & $48(55.2 \%)$ & $30(53.6 \%)$ & $18(58.0 \%)$ \\
\hline 85 - 89 años & $33(37.9 \%)$ & $20(35.7 \%)$ & $13(41.9 \%)$ \\
\hline 90 años a más & $6(6.9 \%)$ & $6(10.7 \%)$ & $0(0 \%)$ \\
\hline Hipercolesterolemia & $220(22.7 \%)$ & $81(36.8 \%)$ & $139(63.2 \%)$ \\
\hline 80 - 84 años & $144(65.5 \%)$ & $55(67.9 \%)$ & $89(64.0 \%)$ \\
\hline 85 - 89 años & $56(25.5 \%)$ & $19(23.5 \%)$ & $37(26.6 \%)$ \\
\hline 90 años a más & $20(9.0 \%)$ & $7(8.6 \%)$ & $13(9.4 \%)$ \\
\hline Diabetes* & $111(11.5 \%)$ & $35(31.5 \%)$ & $76(68.5 \%)$ \\
\hline 80 - 84 años & $67(60.4 \%)$ & $21(8.33 \%)$ & $46(13.73 \%)$ \\
\hline 85 - 89 años & $33(29.8 \%)$ & $11(10.47 \%)$ & $22(13.02 \%)$ \\
\hline 90 años a más & $11(9.8 \%)$ & $3(6.12 \%)$ & $8(13.56 \%)$ \\
\hline IMC $<25$ & $537(55.4 \%)$ & $242(45.0 \%)$ & $295(54.9 \%)$ \\
\hline 80 - 84 años & $302(56.2 \%)$ & $132(54.5 \%)$ & $170(57.6 \%)$ \\
\hline 84 - 89 años & $163(30.4 \%)$ & $69(28.5 \%)$ & $94(31.8 \%)$ \\
\hline 90 años a más & $72(13.4 \%)$ & $41(16.9 \%)$ & $31(10.5 \%)$ \\
\hline IMC 25 - 29.9 & $328(33.8 \%)$ & $144(43.9 \%)$ & $184(56.1 \%)$ \\
\hline 80 - 84 años & $213(64.9 \%)$ & $102(70.8 \%)$ & $111(60.3 \%)$ \\
\hline 85 - 89 años & $84(25.6 \%)$ & $26(18.1 \%)$ & $58(31.5 \%)$ \\
\hline 90 años a más & $31(9.5 \%)$ & $16(11.1 \%)$ & $15(8.2 \%)$ \\
\hline $\mathrm{IMC}>30^{*}$ & $104(10.7 \%)$ & $31(29.8 \%)$ & $73(70.2 \%)$ \\
\hline 80 - 84 años & $71(68.3 \%)$ & $19(61.3 \%)$ & $52(71.2 \%)$ \\
\hline 85 - 89 años & 27 (25.9\%) & $10(32.3 \%)$ & $17(23.3 \%)$ \\
\hline 90 años a más & $6(5.8 \%)$ & $2(6.5 \%)$ & $4(5.5 \%)$ \\
\hline
\end{tabular}


Considerando el nivel de presión y el tratamiento, el $87 \%$ se encontraba con tratamiento farmacológico, de los cuales el $65 \%$ estaban controlados, y un $34 \%$ a pesar del tratamiento, no alcanzaba los rangos normales.

En el grupo de hipertensos que no utilizaban antihipertensivos un $56 \%$ estaba controlando su presión con hábito higiénico dietético y un $43,64 \%$ no alcanzaba las metas respectivas. Tabla 3.

Tabla 3. Distribución por nivel de presión arterial, tratamiento y género

\begin{tabular}{llll} 
& & \multicolumn{2}{c}{ Género (\%) } \\
& Mujeres & Hombres & Total \\
\hline Con tratamiento & 142 & 230 & 372 \\
\hline Controlados & 91 & 152 & 243 \\
\hline No controlados & 51 & 78 & 129 \\
\hline Sin tratamiento & 19 & 36 & 55 \\
\hline Controladas & 10 & 21 & 31 \\
\hline No controladas & $9(47.4 \%)$ & $15(41.7 \%)$ & $24(43.64 \%)$ \\
\hline
\end{tabular}

En la población hipertensa, el 10,07\% eran fumadores, siendo más frecuente en los hombres, con significancia estadística (Figura 2).

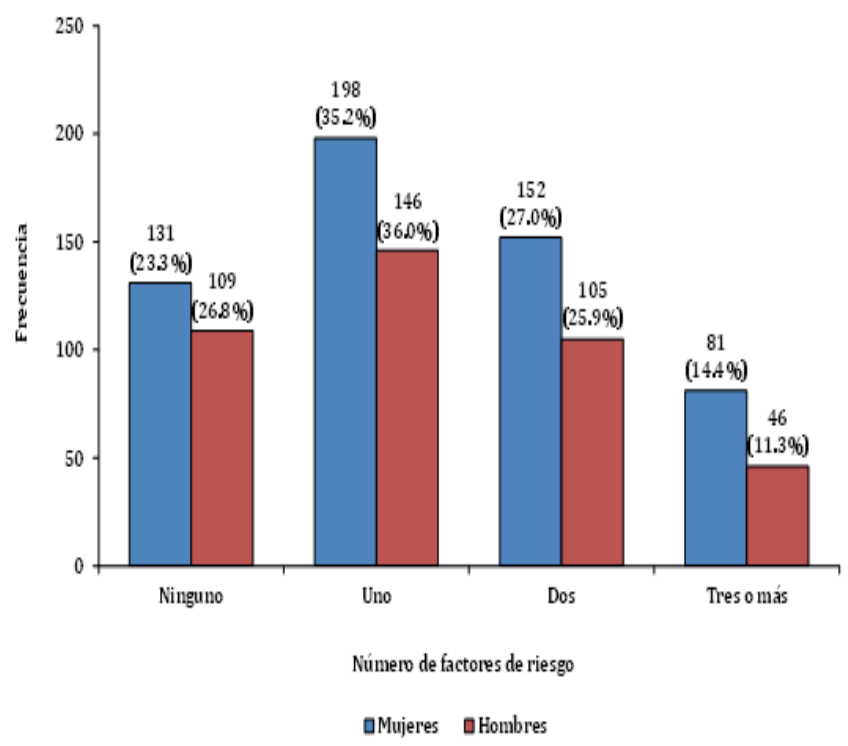

Figura 2. Distribución por número de factores de riesgo según género.
El 33\% tenían colesterol elevado, no existiendo diferencia por género. La diabetes prevalecía en un $16,63 \%$ y un $14,29 \%$ eran obesos, estos dos factores era más prevalente en las mujeres, con significancia estadística. Tabla 4.

Tabla 4. Distribución por factores de riesgo según género en pacientes con antecedentes de hipertensión arterial.

\begin{tabular}{llcl} 
& \multicolumn{2}{c}{ Género (\%) } & Total \\
& Mujeres & Hombres & p \\
\hline Factores de riesgo & & & \\
Fumador & 12 & 31 & 0.000001 \\
Colesterol elevado & 90 & 54 & 0.95 \\
\hline Diabetes & 53 & 18 & 0.019 \\
\hline Obesidad & $48(18.0)$ & $13(8.1)$ & 0.004 \\
\hline
\end{tabular}

\section{DISCUSIÓN}

El concepto de Factor de Riesgo constituye un avance importante en el marco de las estrategias en prevención de las enfermedades cardiovasculares, teniendo en cuenta que desde el punto de vista epidemiológico un factor de riesgo es un predictor estadístico de una enfermedad.

Gracias al estudio del corazón de Framingham y a su director el Dr. William B. Kannel (10), quien utilizó por primera vez el término "Factor de Riesgo", es que en la actualidad reconocemos que la enfermedad coronaria isquémica es multifactorial y es consecuencia de diversos factores de riesgo, muchos de ellos prevenibles.

El Perú ha experimentado una transición epidemiológica fundamental, en donde las enfermedades infecciosas han sido desplazadas como principal causa de mortalidad por las enfermedades crónicas no transmisibles. Por otro lado la expectativa de vida del poblador peruano que en 1990 era de 67 años, veinte años 
después llegó a 74,81 años (77 en mujeres y 72 en varones), la pirámide poblacional muestra que nuestra población longeva crece, y en particular los octogenarios que en el año 2006 alcanzaron la cifra de 450,541 pasaron a ser 609,651 en el 2010, lo que significa un incremento promedio anual de $5,8 \%$.

Y esta población del adulto mayor que está expuesto a los denominados factores de riesgo coronario desarrolla sendas complicaciones cardiovasculares que conllevan a la muerte. Realizar estrategias que permitan reducir esta morbi-mortalidad obliga primero a analizar la situación actual.

El presente estudio realizado en una población mayor de 80 años, demuestra -acorde con la literatura mundial- que la mujer tiene una mayor supervivencia en relación al varón, según los reportes del Instituto Nacional de Estadística e Informática (INEI) las mujeres representan el 58,4\% de la población octogenaria, como consecuencia a un mayor acceso a los sistemas de salud y a mejores condiciones de vida (11).

Pero si bien en los países desarrollados el incremento de la longevidad está acompañado de un aumento de la esperanza de vida sana y de la comprensión de la morbilidad, producto de la mejora sustancial de la calidad de vida de la población; en los países de Latino-América cuando van alcanzando mayor longevidad generalmente padecen una serie de limitaciones de sus funciones producto de presentar enfermedades crónicas, muchas de ellas prevenibles.

Es necesario tener en consideración que la longevidad no es sólo un indicador del envejecimiento poblacional, pues ello implica contar con sistemas de salud lo suficientemente preparados para atender dicha población y no caer en un abandono sanitario; en el estudio actual se ha registrado un significativo $11 \%$ de sujetos mayores de 90 años, evidenciando la tendencia demográfica del país que caracterizará al siglo XXI y que debe obligar a desarrollar futuros programas sociales de atención acorde con esta larga supervivencia.

La principal causa de muerte en la población adulta mayor son las ECV, se estima que cada año la enfermedad cardiovascular ocasiona alrededor de 4 millones de muertes en Europa (47\% de todas las muertes) y 1,9 millones en la Unión Europea (40\%) (12). En USA entre los ancianos en el 2010 hubo cerca de 500 mil muertes por enfermedades del corazón $(26,5 \%)$.

Un número de factores, incluyendo la hipertensión arterial, la hipercolesterolemia, el tabaquismo, la diabetes, el sobrepeso o la obesidad y la inactividad física; favorece que los ancianos sean particularmente vulnerables y aumenten su riesgo de morir por enfermedades del corazón (13).

La HTA es la enfermedad que en mayor medida contribuye al infarto agudo de miocardio y al accidente cerebrovascular. En el registro RENIMA II (14) el $71 \%$ de los infartados eran hipertensos $(68,8 \%$ en varones y $76,5 \%$ en mujeres); en los estudios TORNASOL (15), se incrementó la HTA en la población mayor de 80 años de 47,1\% a 56,3\% (2004 a 2010); y el INEI registra octogenarios hipertensos en un $46,3 \%(13)$.

En el presente estudio, el 44\% era hipertenso y se debe considerar que la HTA constituye un factor de riesgo cardiovascular independiente y sobre todo modificable que exige a tomar medidas preventivas y mejorar el tratamiento del mismo.

El tabaquismo es el único factor de riesgo modificable que en el Perú se ha logrado reducir de $26,1 \%$ a $23,2 \%$, según los estudios TORNASOL; sin embargo en octogenarios no se ha observado esta tendencia; lo cual demanda mejores estrategias antitabaco en este grupo poblacional.

La hipercolesterolemia constituye otro factor de riesgo, que se mantiene inclusive en el adulto mayor y su prevalencia en mayores de 80 años tiende a elevarse como lo demuestran los estudios TORNASOL de $7,6 \%$ a $14,6 \%$; o en el reporte del INEI con una frecuencia de 14,9\%. La evidencia científica obliga a que este factor sea controlado no sólo farmacológicamente sino fundamentalmente con cambios en la dieta.

La diabetes actualmente es uno de los factores de riesgo con mayor crecimiento en la población 
adulta y es consecuencia principalmente del sedentarismo, de una dieta hipercalórica que propician la obesidad, síndrome metabólico y finalmente pueden desarrollar diabetes.

En el RENIMA II la diabetes estuvo presente en un $33,1 \%$ de los sujetos infartados; y en el estudio TORNASOL II la prevalencia en mayores de 80 años era de $5,3 \%$, pero considerando que sólo la mitad conoce su diagnóstico, la cifra real estaría en el orden del 10,6\%; el INEI registra la cifra de 9,2\%; nuestros resultados son similares.

Realizar políticas de salud en el país para evitar la diabetes es cada vez más una necesidad, considerando la experiencia sufrida por esta entidad nosológica en otros países.

La obesidad se ha convertido en varios países desarrollados en una epidemia y se están realizando esfuerzos en los cambios de estilo de vida a fin de reducir su prevalencia.

En el Perú su prevalencia en octogenarios es del orden del 11,9\%; en el estudio INTERHEART sobre factores de riesgo para infarto cardiaco en Latinoamérica, mostró que la obesidad era el primer factor de riesgo. Una reducción de $10 \mathrm{Kg}$ en un año está asociado a una reducción de glicemia de 1,6 $\mathrm{mmol} / \mathrm{L}$, una reducción del $21 \%$ de complicaciones de la diabetes y de un $25 \%$ de la mortalidad por diabetes (16). Por lo que el control del peso y la reducción de la obesidad se debería estimular y fortalecer a través de la educación aún en este grupo etario.

En los sujetos hipertensos del presente estudio se identificó que recibían tratamiento farmacológico en un alto porcentaje (87\%), quizás se explique esta característica por el tipo de población y el acceso a salud que pueden tener al vivir en una ciudad 0 al cuidado de la familia, lo que permite evidenciar el mejor control de la presión arterial, considerando que en el Perú actualmente sólo se controla adecuadamente al 20\%. En el estudio HYVET, clásico estudio en población mayor de 80 años sobre HTA, se observó que el $65 \%$ de la población estudiada se encontraba en tratamiento (17).
Finalmente, la literatura científica muestra que la población cuando desarrolla un evento cardiovascular grave o fatal es portadora de tres 0 más factores de riesgo. En la población estudiada el $40 \%$ se caracterizaba por tener dos o más factores de riesgo. Lo que el profesional de la salud debe evaluar al paciente y estratificar su riesgo según las diversas tablas existentes (Framingham, SCORE europeo, REGICOR, PROCAM, etc.) y establecer programas integrales que busque abordar los diferentes factores de riesgo en forma conjunta.

En conclusión, el factor de riesgo cardiovascular más frecuente en la población estudiada mayor de 80 años ha sido la Hipertensión Arterial, predominando en las mujeres.

El 40\% de los sujetos evaluados se caracterizaban por tener dos o más factores de riesgo cardiovascular.

El $87 \%$ de los pacientes hipertensos recibían tratamiento farmacológico y el 65\% de ellos tenían la presión controlada.

Se recomienda, que el Estado cree una Unidad de Estudios y Vigilancia de las Enfermedades Crónicas no Transmisibles que permita evaluar los factores de riesgo a fin de plantear programas preventivopromocionales que busquen reducir la morbimortalidad cardiovascular.

\section{Agradecimiento}

Al Bachiller en estadística Sr. Raúl Mantilla Quispe, por su asistencia estadística.

\section{Fuentes de financiamiento}

Este artículo ha sido financiado por el autor.

\section{Conflictos de interés}

Los autores declaran no tener conflictos de interés. 


\section{REFERENCIAS BIBLIOGRÁFICAS}

1. Jackson CF, Wenger N. Enfermedad Cardiovascular en el anciano. Rev Esp Cardiol. 2011;64:697-712.

2. INEI. Perú: Estimación y Proyecciones de Población Departamental, por Años Calendario y Edades Simples 1995-2025. Boletín Especial $N^{\circ}$ 22. Lima, Noviembre 2010.

3. INEI. Estado de la Población Peruana 2014. Lima, 2015.

4. American Heart Association. Statistical Fact Sheet 2013 Update. Older Americans \& Cardiovascular Diseases. AHA 2013.

5. Ruiz-Mori E. Riesgo y Prevención Cardiovascular. $1^{\circ}$ Ed. Lima, 2014.

6. Ruiz-Mori E, Segura L, Agusti R. Riesgo cardiovascular y edad vascular según el score de Framingham en el Perú. Diagnóstico, 2013; 52(4):178-186.

7. INEI. La Mortalidad en el Perú. Lima, 2009.

8. Chobanian A. V, Bakris G. L, Black H. R., Cushman W. C., Green L. A, IzzoJ. L. Jr., et al. Joint National Committee on Prevention, Detection, Evaluation, and Treatment of High Blood Pressure; National Heart, Lung, and Blood Institute; National High Blood Pressure Education Program Coordinating Committee. «Seventh Report of the Joint National Committee on Prevention, Detection, Evaluation, and Treatment of High Blood Pressure». Hypertension 2003, 42: 1206-1252.

9. Consenso SEEDO 2000 para la evaluación del sobrepeso y la obesidad y el establecimiento de criterios de intervención terapéutica. Med. Clin. Barc. 2000; 115: 587-597).
10. Romero T. Factores modificables de riesgo cardiovascular: ¿Cuáles estamos realmente modificando? Rev Méd Chile. 2009; 137: 14981501

11. Huenchuan S, Fassio A, Carlos S, Osorio P, Miño A, Batthyany K, y col. Envejecimiento, género y políticas públicas. Lucida Ediciones. Uruguay. 2010.

12. Ferreira-González I. Epidemiología de la enfermedad coronaria. Rev Esp Cardiol. 2014;67:139-144

13. Centers for Disease Control and Prevention, National Center for Health Statistics. Multiple Cause of Death 1999-2010 on CDC WONDER Online Database released 2012. Data are from the Multiple Cause of Death Files, 1999-2010.

14. Reyes M, Ruiz-Mori E. Registro Nacional de Infarto de Miocardio Agudo. RENIMA II. Rev Per Cardiol.2013;39:60-71.

15. Segura L, Agusti R, Ruiz-Mori E. Factores de Riesgo de las enfermedades cardiovasculares en el Perú. TORNASOL II. Rev Per Cardiol.2013;39:5-59.

16. Lanas F. Factores de riesgo cardiovascular en América Latina: estudio INTERHEART. Circulation. 2007;115:1067-1074.

17. Beckett N, Peters R, Fletcher A, Staessen J, Liu L, Dumitrascu D, et al. Treatment of Hypertension in Patients 80 Years of Age or Older. N Engl J Med. 2008;358:1887-1898.

\section{Correspondencia:}

Carlos Enrique Ruiz Mori

Dirección: Angamos Este 2520, Surquillo 15038

Teléfono: 998788580

Correo: cruiz@inen.sld.pe 